ISSN 0258-7122

Bangladesh J. Agril. Res. 36(4): 605-621, December 2011

\title{
CHARACTERIZATION AND MAINTENANCE OF YAM (Dioscorea spp.) GERMPLASM
}

\author{
MD. TARIQUL ISLAM ${ }^{1}$, RAIS UDDIN CHOWDHURY ${ }^{2}$, ROZINA AFROZ ${ }^{3}$ \\ SAJIA RAHMAN ${ }^{4}$ AND MD. MAMTAZUL HAQUE ${ }^{5}$
}

\begin{abstract}
Three experiments were conducted with 60 germplasm accessions of yam (Dioscorea spp.) at Plant Genetic Resources Centre of BARI for characterization and conservation of germplasm. Fifty-nine germplasm accessions of D. alata L. and one accession of D. bulbifera L. from different districts of Bangladesh were used. Clockwise twining direction was found in D. bulbifera and anti-clockwise twining direction was exhibited in all D. alata germplasm accessions. Potato like aerial tuber was found in D. bulbifera. Round, oval, elongate, flattened to irregular aerial tuber shape were found. Grayed-brown, brown to grayed-orange skin colour with yellow, yellow-orange to grayed-orange of aerial tuber flesh colour were observed among the yam germplasm accessions. Oval-oblong, cylindrical, flattened, corm, rhizome to irregular shape of underground stem or tubers were observed. Low, medium to dense types of spiniess of roots were found on the tubers. Grayed-orange, red to black tuber skin colour along with white, yellowish-white, yellow, yelloworange, red to purple tuber flesh colours were found. Significant variations were also observed in stem shape at base, colour of stem, wing and petiole wing, position of leaf, leaf shape and distance between leaf lobes. Low to high phenotypic diversity was exhibited among the germplasm accessions. The germplasm accessions produced 1 to 30 under ground tubers per plant. The main tuber length ranged from 5.4 to $66.4 \mathrm{~cm}$ and breadth from 2.5 to $15.5 \mathrm{~cm}$. The germplasm accessions produced $0.51 \mathrm{~kg}$ to $16.45 \mathrm{~kg}$ tuber yield per plant. The maximum variability was found in tuber yield per plant $(98.74 \%)$ and the minimum was found in leaf length (12.55\%).The under ground tubers of yam were harvested and replanted at an interval of two to three years from 1995 at PGRC. The plants were grown on bamboo trail in the field genebank. Necessary intercultural operations are done for maintaining the yam germplasm accessions.
\end{abstract}

Keywords: Dioscorea, characterization, phenotypic diversity, field genebank, Bangladesh.

\section{Introduction}

Yams (Dioscorea spp.) are distributed in the tropical, subtropical and temperate region of the world. There are 8 genera and 880 species of yam (Mabberley, 1997). Yams are underutilized crops in Bangladesh. They are grown almost all

${ }^{1,2 \& 3}$ Senior Scientific Officer, Plant Genetic Resources Centre, Bangladesh Agricultural Research Institute (BARI), Gazipur 1701, ${ }^{4}$ Scientific Officer, Plant Genetic Resources Centre (BARI), Gazipur 1701, ${ }^{5}$ Former Chief Scientific Officer, Plant Genetic Resources Centre (BARI), Gazipur 1701, Bangladesh. 
over the country and used as vegetable. Yam plants produce edible tubers, bulbils, corms or rhizomes. The approximate composition of edible yam tubers is water (65-75\%), carbohydrates, mainly starch (15-25\%), protein (1-2.5\%), fibre (0.5-1.5\%), ash (0.7-2.08\%), and fat (0.05-0.20\%) (Ammirato, 1984). It contains approximately $8-10 \mathrm{mg} / 100 \mathrm{~g}$ of ascorbic acid and most of it is retained during cooking. Vitamin A is present in yellow flesh varieties. Calcium, iron, and phosphorus contents are high among the materials (Ammirato, 1984). The yams are rarely consumed alone. They are normally eaten with meat, fish, green vegetables, coconut, and spices in quantities varying with the taste and resources of the consumer. Yams are the important food crops in many tropical and subtropical countries (Onwueme, 1978). Most advantageous is that this crop can be cultivated even by the side of the roads, homestead gardens, houses and in the jungles, etc. No extra land is needed for yam cultivation like other root and tuber crops, such as potato and sweet potato. Different ethnic groups cultivated yam under shade in hill and forests in Bangladesh. No attempt was made to release any variety considering the marginal economic importance of the crop, which is not even grown on a commercial scale. Before the introduction of potato, the yams were used in this country as common vegetables. This has now been replaced by potato (Zaman, 1989). Systematic work on identification of species and crop improvement programme should be continued on yam, though it has wide use as a vegetable all over the country, and as staple food to some ethnic communities. Local knowledge regarding utilization of crop is needed to document its economic values. Tribals grew yam under shade in hilly regions in forests and have a good harvest (Islam et al., 1997). There is a possibility of introducing this crop in existing cropping patterns enhancing crop diversification programmes in Bangladesh. There are 18 species under Dioscorea so far known in Bangladesh. They are D. alata L. ("Chupri alu", Greater Asiatic yam), D. aculeata L. ("Gointa alu",), D. belophylla Prain ("Shora alu" ), D. bulbifera var. bulbifera ("Pagla alu", Potato yam), D. glabra Roxb. ("Sorg alu"), D. hamiltonii Hooker, D. hispida Dennstedt, D. melanophyma Prain and Burkill, D. oppositifolia L., D. pentaphylla L. ("Jum alu or Kanta alu"), D. prazeri Prain and Burkill, D. pubera Blume ("Kukur alu7), D. bulbifera var. sativa ("Mou alu'), D. esculents Lour. (Lesser yam), D. kamoonensis Kunth. ("Erabera lata"), D. spicata Roth., D. tomentosa Koenig ex Spreng, D. trinervia Roxb ex Prain and Burkill. Most of the species are available in Chittagong, Chittagong Hill Tracts districts, Rangamati, Noakhali, and Sylhet. In addition, some species are recorded from Cox's Bazar, Lakshmipur, Dhaka, Munshiganj, Gazipur, Tangail, Jamalpur, Sherpur, Rajshahi, Rangpur and Satkhera (Rahman et al., 2001; Rahman et al., 1998; Sinclair, 1956; Raizada, 1941; Cowan, 1928; Heinig, 1925; Prain, 1903; Prain, 1903a; Hooker 1892; Roxburgh, 1832 and Roxburgh, 1814). Current nomenclature, common and vernacular names, distributions, first record and ethnobotanical information were recorded by Rahman et al. (2001). Islam et al. 
(1995) conducted an ethnobotanical survey to document local knowledge on the uses and conservation of taros and yam. Four species of yam were identified within 15 districts and among 21 ethnic groups in Bangladesh. The species are $D$. alata, D. bulbifera, D. esculents and D. dumetorum. The districts were Dinajpur, Rangpur, Bogra, Rajshahi, Pabna, Jessore, Khulna, Dhaka, Tangail, Mymensingh, Comilla, Sylhet, Noakhali, Chittagong and Chittagong Hill Tracts.

Proper characterization and evaluation of germplasm and dissemination of the information to the plant breeders and others is a priority area in any germplasm management programme. Characterization of germplasm consists traits that are highly heritable, expressed in different environments and can be easily seen by the eye. Characterization information along with passport data provides an indication of the range of diversity in the collections, and is of considerable help to the breeders to narrow the selection of potential breeding stocks. Until a collection has been properly evaluated and its attributes become known to breeders, it has little practical use (Thomas and Mathur, 1991). The field genebank is a facility where clonal materials like yams are conserved as living collections in field, orchard or in plantation (Chomchalov, 1985). It is one of the ex situ conservation. The advantage of maintaining field genebanks is that the germplasm can be readily characterized and evaluated and as well as conserved. Systematic research, such as collection, characterization, evaluation, and maintenance of yam have been done at PGRC. This article addresses characterization and maintenance of yam in the field genebank. In this context, the present study was undertaken aiming at the following objectives (i) To characterize and identify the promising germplasm accessions for varietal improvement programme, (ii) To maintain the germplasm accessions at the field genebank for future uses by plant breeders and researchers.

\section{Materials and Method}

Three experiments were conducted with 60 germplasm accessions of yam (Dioscorea spp.) at Plant Genetic Resources Centre of BARI, Gazipur during 1998 to 2006. Forty-two germplasm accessions were collected from 9 districts, namlely Dinajpur, Rangpur, Bogra, Jessore, Mymensingh, Dhaka, Comilla, Chittagong, and Sylhet of Bangladesh on ethnobotanical survey on taro and yam during 1994-95. The remaining germplasm accessions were collected from Rajshahi, Pabna, Chittagong, and Cox's Bazar in 2001. The first, second, and third experiments were planted on 10 April 1998, 15 April 2002, and 27 April 2004 with 42, 60, and 60 germplasm accessions, respectively. Plant to plant spacing of first, second, and third experiment was $3 \mathrm{~m}, 2 \mathrm{~m}$, and $4 \mathrm{~m}$, respectively. Aerial and under ground tubers were used. Three plants were grown from each accession per plot of 3 pits. Approximately, $10 \mathrm{~kg}$ cowdung, $100 \mathrm{~g}$ urea, $150 \mathrm{~g}$ 
triple super phosphate and $120 \mathrm{~g}$ murate of potash were used in each pit as basal doses. Sufficient loamy soil was added in the pit. The experiment was laid out in a non replicated design. The plants were grown on bamboo trail. All intercultural operations were followed to have a good crop. The aerial and under ground tubers were collected in the month of March 2001, 2004, and 2006 from the first, second, and third experiment, respectively. Different observations were recorded as per Descriptors for Yam (IPGRI, 1997). Chi-square $\left(\chi^{2}\right)$ test was done for each qualitative character. Phenotypic diversity for qualitative traits are determined by using Shannon-Weaver Diversity Index $\left(\mathrm{H}^{\prime}\right)$. $\mathrm{H}^{\prime}$ ranges from 0 to 1 , where 1 indicates the maximum diversity ( $\mathrm{Yu} \mathrm{Li}$ et al., 1996). $\mathrm{H}^{\prime}$ is defined as: $\mathrm{H}^{\prime}=$ $\Sigma \mathrm{PiLOg}{ }_{2} \mathrm{Pi}$, where $\mathrm{Pi}$ is the proportion of the total number of germplasm accessions belonging to ith class. $\mathrm{H}^{\prime}$ (SWDI) classified as low $\left(\mathrm{H}^{\prime}<0.50\right)$, medium or intermediate $\left(H^{\prime}=0.50-0.75\right)$ and high $\left(H^{\prime} \geq 0.75\right)$ based on Jamago (2000). Range, mean, standard deviation and coefficient of variation for different quantitative characters are calculated.

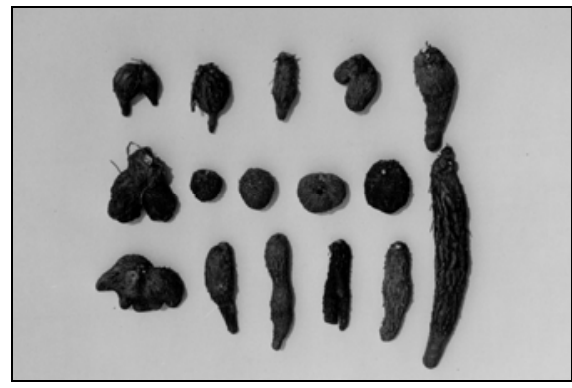

Fig. 1. Aerial tuber diversity in 1998-01

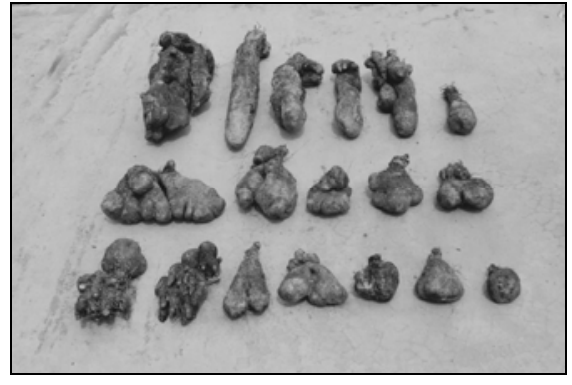

Fig. 2. Aerial tuber diversity in 2004-06 Upper row - BD-7866 (3 branch), BD7847, BD-7872, BD-7857, BD-7847, BD7852 (Left to Right)- ( Total-6) $1^{\text {st }}$ middle row - BD-7850, BD-7882, BD7851, BD-7859, BD-7868 (Total-5)

$2^{\text {nd }}$ middle row - BD-7861, BD-7849, BD7897, BD-7883, BD-7877, BD-7865 (Total-6)

Lower low- BD-7891, BD-7898, BD-7841, BD-7880, BD-7896, BD-7893 (Total-6) 


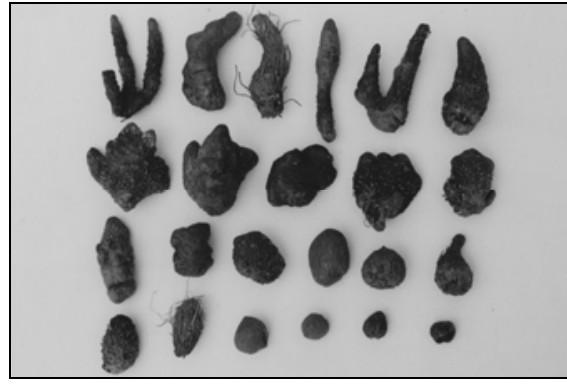

Fig. 3. Underground tuber diversity-2002-04

Upper row - BD-7866 (largest), BD-7889, 1313-7867,1313-7883, BD-7879, BD-7887 (Left to Right)-(Total-6)

Middle row- BD-7894, HOM-3, BD-7898 , BD-7854, BD-7887(Total-5)

Lower low- BD-7861 , BD-7851, BD7857, BD-7896 , BD-7850 , BD-7859, BD-7880 (Total-7)

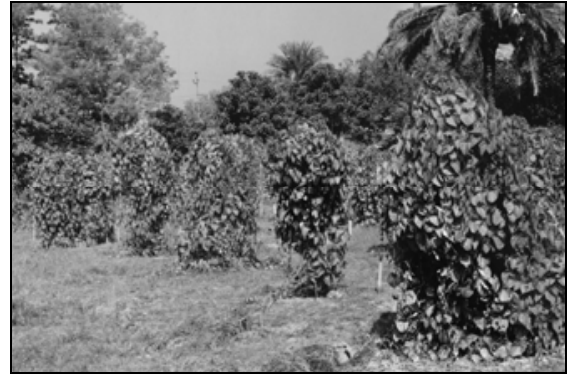

Fig. 4. Maintenance of yam germplasm accessions at the field genebank, PGRC, BARI, Gazipur

Figure legends: Maintenance of yam (Dioscorea spp.) at PGRC, BARI, 1994 to 2006.

\section{Results and Discussion}

Among the three experiments, 59 germplasm accessions were identified as D. alata and the rest one was D. bulbifera (BD-7838). The tubers were sprouted and produced aerial part after monsoon rain during April and May. The accession of D. bulbifera climbed the bamboo to the left (clockwise). In first experiment (1998-01), green to purplish-green stem with 4 and 7 ridge was found at the base of stem. Colour of stem wing was purple to green with purple edge. Colour of petiole was green, all green with purple base and all green with purple at both ends; and colour of petiole wing was green to purple. The germplasm accessions exhibited alternate, opposite, and alternate at base and opposite above in leaf position. Almost all the characters showed highly significant differences (Table la). Cordate to sagittate leaf shape along with no measurable distance to intermediate distance between lobes were found among the germplasm accessions. Aerial tuber was found in 32 germplasm accessions and 10 germplasm accessions did not produce any aerial tuber. The germplasm accessions exhibited round, oval, elongate, flattened to irregular aerial tuber shape with grayed-brown to brown tuber skin colour, and wrinkled to rough surface texture. Highly significant variations were found among the germplasm accessions. Bumps were found in all the aerial tubers (Fig.1 and Table lb). All 
Table la. Qualitative variations of different characters in yam at PGRC, 1998-01.

\begin{tabular}{|c|c|c|c|c|c|c|c|c|c|c|c|}
\hline $\begin{array}{l}\text { Stem shape } \\
\text { at base }\end{array}$ & No. of Acc. & $\begin{array}{l}\text { Stem } \\
\text { colour }\end{array}$ & No. of Acc & $\begin{array}{l}\text { Colour of } \\
\text { stem wing }\end{array}$ & No. of Acc. & $\begin{array}{l}\text { Petiole } \\
\text { colour }\end{array}$ & $\begin{array}{l}\text { No. of } \\
\text { Acc. }\end{array}$ & $\begin{array}{c}\text { Position of } \\
\text { leaf }\end{array}$ & No. of Acc. & $\begin{array}{c}\text { Petiole } \\
\text { wing } \\
\text { colour }\end{array}$ & No. of Acc \\
\hline Ridge (7) & 3 & Green & 37 & Purple & 7 & GPB & 20 & Alternate & 2 & Green & 4 \\
\hline \multirow[t]{2}{*}{ Ridge (4) } & 39 & $\begin{array}{l}\text { Purplish- } \\
\text { green }\end{array}$ & 5 & GPE & 35 & GPE & 14 & Opposite & 2 & Purple & 38 \\
\hline & & & & & & Green & 8 & $\mathrm{AO}$ & 38 & & \\
\hline$\chi^{2}$ value & 30.86 & & 24.38 & & 18.67 & & 5.14 & & 61.71 & & 27.52 \\
\hline $\begin{array}{l}\text { Level of } \\
\text { significance }\end{array}$ & $* *$ & & $* *$ & & $* *$ & & $* *$ & & $* *$ & & $* *$ \\
\hline SWDI & $\begin{array}{l}0.37 \\
(\mathrm{~L})\end{array}$ & & $\begin{array}{l}0.53 \\
(\mathrm{M})\end{array}$ & & $\begin{array}{l}0.65 \\
(\mathrm{M})\end{array}$ & & $\begin{array}{l}0.94 \\
(\mathrm{H})\end{array}$ & & $\begin{array}{l}0.35 \\
(\mathrm{~L})\end{array}$ & & $\begin{array}{l}0.45 \\
(\mathrm{~L})\end{array}$ \\
\hline
\end{tabular}

Where, GPE-Green with edge; GPB-All green with purple base, GPE-All green with purple at both ends; AO-Alternate at base and oppsite above; SWDI-Shannon-Weaver Diversity Index, H-High, M-Moderate, L-Low; ns-not significant, *-Significant at $5 \%$ and ${ }^{* *}$-Significant at $1 \%$ probability.

Table lb. Qualitative variations of different characters in yam at PGRC, 1998-01.

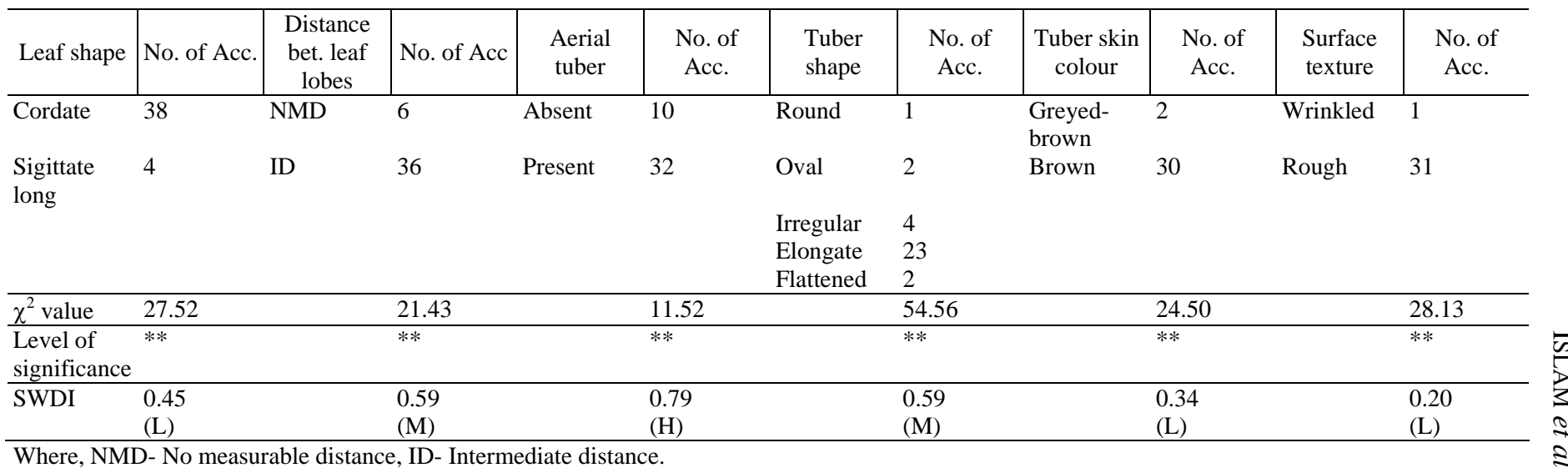


Table Ic. Qualitatively no variations of different characters in yam at PGRC, 1998-01.

\begin{tabular}{|c|c|c|c|c|c|c|c|c|c|c|c|}
\hline $\begin{array}{l}\text { Twining } \\
\text { direction }\end{array}$ & No. of Acc. & $\begin{array}{c}\text { Wing } \\
\text { position on } \\
\text { stem }\end{array}$ & No. of Acc. & Leaf colour II & No. of Acc. & $\begin{array}{l}\text { Leaf } \\
\text { margin } \\
\text { colour }\end{array}$ & No. of Acc. & $\begin{array}{l}\text { Vein } \\
\text { colour }\end{array}$ & No. of Acc. & $\begin{array}{c}\text { Presence of } \\
\text { bumps on } \\
\text { aerial }\end{array}$ & $\begin{array}{l}\text { No. of } \\
\text { Acc. }\end{array}$ \\
\hline Anticlock & 42 & $\begin{array}{l}\text { All over } \\
\text { stem }\end{array}$ & 42 & Green & 42 & Green & 42 & Green & 42 & Present & 32 \\
\hline Aerial shape & $\begin{array}{c}\text { No. of } \\
\text { Acc. }\end{array}$ & $\begin{array}{c}\text { Aerial } \\
\text { tuber skin } \\
\text { colour }\end{array}$ & $\begin{array}{l}\text { No. of } \\
\text { Acc. }\end{array}$ & $\begin{array}{c}\text { Aerial tube } \\
\text { skin } \\
\text { thickness }\end{array}$ & $\begin{array}{l}\text { No. of } \\
\text { Acc. }\end{array}$ & $\begin{array}{l}\text { Aerial tuber } \\
\text { flesh colour }\end{array}$ & $\begin{array}{l}\text { No. of } \\
\text { Acc. }\end{array}$ & $\begin{array}{c}\text { Aerial } \\
\text { tuber } \\
\text { surface } \\
\text { texture }\end{array}$ & $\begin{array}{l}\text { No. of } \\
\text { Acc. }\end{array}$ & $\begin{array}{l}\text { Presence of } \\
\text { bumps on } \\
\text { aerial tuber }\end{array}$ & $\begin{array}{c}\text { No. of } \\
\text { Acc. }\end{array}$ \\
\hline Elongate & 36 & $\begin{array}{l}\text { Grayed- } \\
\text { orange }\end{array}$ & 45 & Thin & 27 & Yellow & 27 & Rough & 48 & Present & 29 \\
\hline \multirow[t]{2}{*}{ Irregular } & 14 & $\begin{array}{l}\text { Grayed- } \\
\text { brown }\end{array}$ & 15 & Thick & 23 & $\begin{array}{l}\text { Yellow } \\
\text { orange }\end{array}$ & 19 & Smooth & 2 & Absent & 21 \\
\hline & & & & & & $\begin{array}{l}\text { Grayed- } \\
\text { orange }\end{array}$ & 4 & & & & \\
\hline$\chi^{2}$ value & 9.68 & & 15.00 & & 0.32 & & 16.36 & & 42.32 & & 1.28 \\
\hline $\begin{array}{l}\text { Level of } \\
\text { significance }\end{array}$ & $* *$ & & $* *$ & & $* *$ & & $* *$ & & $* *$ & & $* *$ \\
\hline SWDI & $\begin{array}{l}0.86 \\
(\mathrm{H})\end{array}$ & & $\begin{array}{l}0.81 \\
(\mathrm{H})\end{array}$ & & $\begin{array}{l}0.99 \\
(\mathrm{H})\end{array}$ & & $\begin{array}{l}0.82 \\
(\mathrm{H})\end{array}$ & & $\begin{array}{l}0.24 \\
(\mathrm{~L})\end{array}$ & & $\begin{array}{l}0.98 \\
(\mathrm{~L})\end{array}$ \\
\hline
\end{tabular}


Table 3. Qualitative variations of different characters in yam at PGRC, 2002-04.

\begin{tabular}{|c|c|c|c|c|c|c|c|c|c|c|c|}
\hline $\begin{array}{l}\text { Twining } \\
\text { direction }\end{array}$ & $\begin{array}{c}\text { No. of } \\
\text { Acc. }\end{array}$ & $\begin{array}{c}\text { Type of } \\
\text { under } \\
\text { ground } \\
\text { stem }\end{array}$ & $\begin{array}{l}\text { No. of } \\
\text { Acc. }\end{array}$ & Tuber shape & $\begin{array}{l}\text { No. of } \\
\text { Acc. }\end{array}$ & $\begin{array}{c}\text { Spininess } \\
\text { on tuber }\end{array}$ & $\begin{array}{l}\text { No. of } \\
\text { Acc. }\end{array}$ & $\begin{array}{l}\text { Tuber skin } \\
\text { colour }\end{array}$ & $\begin{array}{l}\text { No. of } \\
\text { Acc. }\end{array}$ & $\begin{array}{c}\text { Tuber flesh } \\
\text { colour }\end{array}$ & $\begin{array}{c}\text { No. of } \\
\text { Acc. }\end{array}$ \\
\hline Clockwise & 1 & Corm & 2 & Irregular & 2 & Low & 23 & $\begin{array}{l}\text { Grayed- } \\
\text { orange }\end{array}$ & 51 & Purple & 4 \\
\hline \multirow{3}{*}{$\begin{array}{l}\text { Anti- } \\
\text { clockwise }\end{array}$} & 59 & Tuber & 58 & Cylindrical & 47 & Medium & 28 & Red & 8 & White & 22 \\
\hline & & & & Flattened & 8 & Dense & 9 & Black & 1 & $\begin{array}{l}\text { Yellow- } \\
\text { white }\end{array}$ & 34 \\
\hline & & & & Oval oblong & 3 & & & & & & \\
\hline$\chi^{2}$ value & 56.07 & & 52.27 & & 92.40 & & 9.70 & & 73.30 & & 22.80 \\
\hline $\begin{array}{l}\text { Level of } \\
\text { significance }\end{array}$ & $* *$ & & $* *$ & & $* *$ & & $* *$ & & $* *$ & & $* *$ \\
\hline SWDI & $\begin{array}{l}0.12 \\
(\mathrm{~L})\end{array}$ & & $\begin{array}{l}0.21 \\
(\mathrm{~L})\end{array}$ & & $\begin{array}{l}0.52 \\
(\mathrm{M})\end{array}$ & & $\begin{array}{l}0.92 \\
(\mathrm{H})\end{array}$ & & $\begin{array}{l}0.43 \\
(\mathrm{~L})\end{array}$ & & $\begin{array}{l}0.79 \\
(\mathrm{~L})\end{array}$ \\
\hline
\end{tabular}


Table 4. Qualitative variation of under-ground tuber in yam at PGRC, 2004-06.

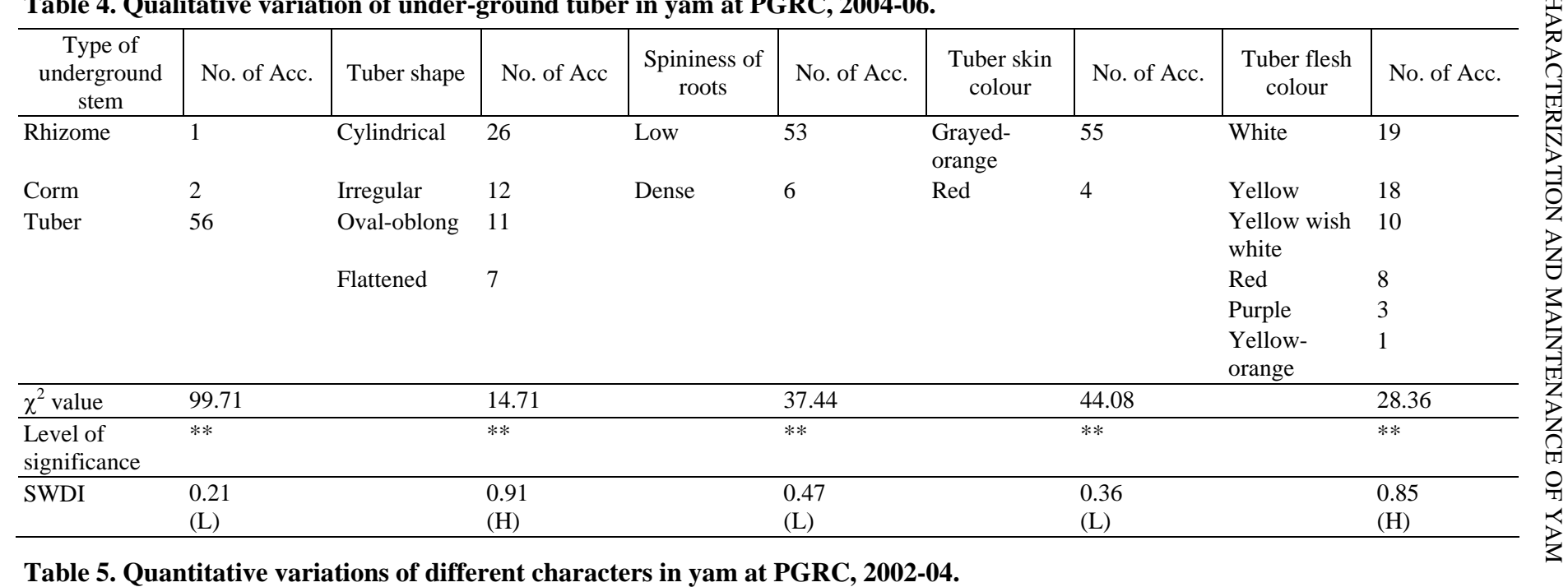

Table 5. Quantitative variations of different characters in yam at PGRC, 2002-04.

\begin{tabular}{l|l|l|l|c|c}
\hline \multicolumn{1}{c}{ Name of descriptor } & \multicolumn{2}{c|}{$\begin{array}{c}\text { No. of germplasm } \\
\text { accessions }\end{array}$} & \multicolumn{1}{c|}{ Range } & Mean & SD \\
\hline Leaf length (cm) & 60 & $11.86-23.14$ & 18.56 & 2.33 & 12.55 \\
Leaf width (cm) & 60 & $6.86-15.79$ & 12.01 & 1.84 & 15.33 \\
Number of tubers per plant & 60 & $1 .-30$ & 9.48 & 7.85 & 82.81 \\
Length of main tuber (cm) & 60 & $5.4-66.4$ & 31.14 & 12.30 & 39.52 \\
Width of main tuber (cm) & 60 & $2.5-15.5$ & 8.18 & 2.41 & 29.41 \\
Under ground tuber yield (kg) & 60 & $0.51-16.45$ & 4.54 & 4.48 & 98.74 \\
\hline
\end{tabular}


Table 6. Listing different characters in yam at PGRC, 1998-01.

\begin{tabular}{|c|c|c|c|c|c|c|c|c|c|c|c|c|c|c|}
\hline $\begin{array}{c}\text { Accession } \\
\text { number }\end{array}$ & $\begin{array}{l}\text { Collectors } \\
\text { number }\end{array}$ & $\begin{array}{l}\text { Stem } \\
\text { shape } \\
\text { at base }\end{array}$ & $\begin{array}{l}\text { Stem } \\
\text { colour }\end{array}$ & $\begin{array}{l}\text { Wing } \\
\text { colour }\end{array}$ & $\begin{array}{l}\text { Petiole } \\
\text { colour }\end{array}$ & $\begin{array}{c}\text { Position } \\
\text { of leaf }\end{array}$ & $\begin{array}{c}\text { Petiole } \\
\text { wing } \\
\text { colour }\end{array}$ & $\begin{array}{l}\text { Leaf } \\
\text { shape }\end{array}$ & $\begin{array}{c}\text { Distance } \\
\text { between } \\
\text { lobes }\end{array}$ & $\begin{array}{c}\text { Aerial } \\
\text { tuber }\end{array}$ & $\begin{array}{l}\text { Tuber } \\
\text { shape }\end{array}$ & $\begin{array}{l}\text { Tuber } \\
\text { skin } \\
\text { colour }\end{array}$ & $\begin{array}{l}\text { Suface } \\
\text { texture }\end{array}$ & $\begin{array}{l}\text { Presence } \\
\text { of bumps }\end{array}$ \\
\hline 1 & 2 & 3 & 4 & 5 & 6 & 7 & 8 & 9 & 10 & 11 & 12 & 13 & 14 & 15 \\
\hline BD-7840 & HOM-7 & $4 \mathrm{R}$ & $130 \mathrm{C}$ & GP & G & $\mathrm{AO}$ & $\mathrm{P}$ & C & I & $\mathrm{P}$ & E & 200B & $\mathrm{R}$ & $\mathrm{P}$ \\
\hline BD-7841 & HOM-8 & $4 \mathrm{R}$ & PG & GP & GPB & A & $\mathrm{P}$ & $\mathrm{C}$ & I & A & $\mathrm{E}$ & $200 \mathrm{~A}$ & $\mathrm{R}$ & $\mathrm{P}$ \\
\hline BD-7844 & HOM-13 & $4 \mathrm{R}$ & $130 \mathrm{~B}$ & GP & G & $\mathrm{AO}$ & G & C & I & $\mathrm{P}$ & $\mathrm{E}$ & 199A & $\mathrm{R}$ & $\mathrm{P}$ \\
\hline BD-7845 & HOM-17 & $4 \mathrm{R}$ & PG & $75 B$ & GPB & $\mathrm{AO}$ & $\mathrm{P}$ & SL & I & $\mathrm{P}$ & $\mathrm{E}$ & 200D & $\mathrm{R}$ & $\mathrm{P}$ \\
\hline BD-7847 & HOM-20 & $4 \mathrm{R}$ & $130 \mathrm{C}$ & $75 \mathrm{~A}$ & GPB & $\mathrm{AO}$ & $\mathrm{P}$ & SL & I & $\mathrm{P}$ & $\mathrm{E}$ & $200 \mathrm{C}$ & $\mathrm{R}$ & $\mathrm{P}$ \\
\hline BD-7848 & HOM-25 & $4 \mathrm{R}$ & $130 \mathrm{~B}$ & GP & GP & $\mathrm{AO}$ & $\mathrm{P}$ & $\mathrm{C}$ & I & $\mathrm{P}$ & - & - & - & - \\
\hline BD-7856 & HOM-39 & $4 \mathrm{R}$ & $130 \mathrm{C}$ & GP & G & $\mathrm{AO}$ & $\mathrm{P}$ & C & I & A & $\mathrm{E}$ & $200 \mathrm{C}$ & $\mathrm{R}$ & $\mathrm{P}$ \\
\hline BD-7857 & HOM-43 & $4 \mathrm{R}$ & $130 \mathrm{C}$ & GP & G & A & G & SL & I & $\mathrm{P}$ & $\mathrm{E}$ & $200 \mathrm{~A}$ & $\mathrm{R}$ & $\mathrm{P}$ \\
\hline BD-7858 & HOM-46 & $4 \mathrm{R}$ & $130 \mathrm{C}$ & GP & GPB & $\mathrm{AO}$ & $\mathrm{P}$ & C & I & $\mathrm{P}$ & I & 200D & $\mathrm{R}$ & $\mathrm{P}$ \\
\hline BD-7859 & HOM-47 & $4 \mathrm{R}$ & $130 \mathrm{C}$ & GP & GP & $\mathrm{AO}$ & $\mathrm{P}$ & $\mathrm{C}$ & I & $\mathrm{P}$ & $\mathrm{E}$ & $200 \mathrm{C}$ & $\mathrm{R}$ & $\mathrm{P}$ \\
\hline BD-7860 & HOM-48 & $4 \mathrm{R}$ & PG & $75 B$ & GPB & $\mathrm{AO}$ & $\mathrm{P}$ & SL & I & $\mathrm{P}$ & E & 200D & $\mathrm{R}$ & $\mathrm{P}$ \\
\hline BD-7861 & HOM-50 & $4 \mathrm{R}$ & $130 \mathrm{~B}$ & GP & GPB & $\mathrm{AO}$ & $\mathrm{P}$ & C & I & $\mathrm{P}$ & - & - & - & - \\
\hline BD-7862 & HOM-51 & $4 \mathrm{R}$ & $130 \mathrm{C}$ & GP & GP & $\mathrm{AO}$ & $\mathrm{P}$ & C & I & A & $\mathrm{E}$ & $200 \mathrm{C}$ & $\mathrm{R}$ & $\mathrm{P}$ \\
\hline
\end{tabular}


Table 6. Cont'd.

\begin{tabular}{|c|c|c|c|c|c|c|c|c|c|c|c|c|c|c|}
\hline BD-7863 & HOM-52 & $4 \mathrm{R}$ & $130 \mathrm{C}$ & GP & GPB & $\mathrm{AO}$ & $\mathrm{P}$ & SL & I & $\mathrm{P}$ & E & $200 C$ & $\mathrm{R}$ & $\mathrm{P}$ \\
\hline BD-7864 & HOM-53 & $4 \mathrm{R}$ & 130B & GP & GP & $\mathrm{AO}$ & $\mathrm{P}$ & SL & I & $\mathrm{P}$ & $\mathrm{E}$ & $200 \mathrm{C}$ & $\mathrm{R}$ & $\mathrm{P}$ \\
\hline BD-7865 & HOM-56 & $4 \mathrm{R}$ & $130 \mathrm{C}$ & GP & G & 0 & G & SL & I & $\mathrm{P}$ & & - & - & - \\
\hline BD-7867 & HOM-58 & $4 \mathrm{R}$ & $130 \mathrm{C}$ & GP & GP & $\mathrm{AO}$ & $\mathrm{P}$ & C & I & A & I & $200 \mathrm{C}$ & $\mathrm{R}$ & $\mathrm{P}$ \\
\hline BD-7868 & HOM-59 & $7 \mathrm{R}$ & 130B & $75 B$ & GPB & $\mathrm{AO}$ & $\mathrm{P}$ & $\mathrm{C}$ & I & $\mathrm{P}$ & $\mathrm{E}$ & $200 C$ & $\mathrm{R}$ & $\mathrm{P}$ \\
\hline BD-7869 & HOM-60 & $4 \mathrm{R}$ & 130B & GP & GP & $\mathrm{AO}$ & $\mathrm{P}$ & $\mathrm{C}$ & I & $\mathrm{P}$ & E & $200 C$ & $\mathrm{R}$ & $\mathrm{P}$ \\
\hline BD-7871 & HOM-62 & $4 \mathrm{R}$ & 130B & GP & GP & $\mathrm{AO}$ & $\mathrm{P}$ & $\mathrm{C}$ & I & $\mathrm{P}$ & E & $200 C$ & $\mathrm{R}$ & $\mathrm{P}$ \\
\hline BD-7872 & HOM-63 & $4 \mathrm{R}$ & 130B & GP & GP & $\mathrm{AO}$ & $\mathrm{P}$ & SL & I & $\mathrm{P}$ & E & $200 \mathrm{D}$ & $\mathrm{R}$ & $\mathrm{P}$ \\
\hline BD-7873 & HOM-64 & $4 \mathrm{R}$ & 130B & GP & GP & $\mathrm{AO}$ & $\mathrm{P}$ & $\mathrm{C}$ & I & $P$ & - & - & - & - \\
\hline BD-7874 & HOM-65 & $4 \mathrm{R}$ & 130B & GP & GPB & $\mathrm{AO}$ & $\mathrm{P}$ & $\mathrm{C}$ & I & A & - & - & - & - \\
\hline BD-7875 & HOM-66 & $4 \mathrm{R}$ & $130 \mathrm{~B}$ & GP & GP & $\mathrm{AO}$ & $\mathrm{P}$ & $\mathrm{C}$ & $\mathrm{N}$ & A & I & $200 C$ & $\mathrm{R}$ & $\mathrm{P}$ \\
\hline BD-7879 & HOM-70 & $4 \mathrm{R}$ & $130 \mathrm{C}$ & GP & GP & $\mathrm{AO}$ & $\mathrm{P}$ & $\mathrm{C}$ & $\mathrm{N}$ & $\mathrm{P}$ & $\mathrm{R}$ & 200B & $\mathrm{R}$ & $\mathrm{P}$ \\
\hline BD-7880 & HOM-71 & $4 \mathrm{R}$ & $130 \mathrm{C}$ & GP & GPB & $\mathrm{AO}$ & $\mathrm{P}$ & $\mathrm{C}$ & I & $P$ & E & $200 C$ & $\mathrm{R}$ & $\mathrm{P}$ \\
\hline BD-7881 & HOM-72 & $4 \mathrm{R}$ & $130 \mathrm{C}$ & GP & G & $\mathrm{AO}$ & $\mathrm{P}$ & SL & I & $\mathrm{P}$ & $\mathrm{F}$ & 200B & $\mathrm{R}$ & $\mathrm{P}$ \\
\hline BD-7882 & HOM-73 & $7 \mathrm{R}$ & 130B & GP & GPB & $\mathrm{AO}$ & $\mathrm{P}$ & $\mathrm{C}$ & I & $\mathrm{P}$ & E & 200D & $\mathrm{R}$ & $\mathrm{P}$ \\
\hline BD-7883 & HOM-74 & $4 \mathrm{R}$ & 130B & GP & GP & $\mathrm{AO}$ & $\mathrm{P}$ & C & $\mathrm{N}$ & $\mathrm{P}$ & E & 200D & $\mathrm{R}$ & $\mathrm{P}$ \\
\hline BD-7884 & HOM-75 & $7 \mathrm{R}$ & $130 \mathrm{C}$ & GP & GP & $\mathrm{AO}$ & $\mathrm{P}$ & C & I & $\mathrm{P}$ & $\mathrm{F}$ & 200B & W & $\mathrm{P}$ \\
\hline
\end{tabular}


Table 7. Listing different characters in yam at PGRC, 2002-04.

\begin{tabular}{|c|c|c|c|c|c|c|c|c|c|c|c|c|c|}
\hline $\begin{array}{c}\text { Accession } \\
\text { number }\end{array}$ & $\begin{array}{l}\text { Collectors } \\
\text { number }\end{array}$ & $\begin{array}{l}\text { Twining } \\
\text { direction }\end{array}$ & $\begin{array}{c}\text { Type of } \\
\text { underground } \\
\text { tuber }\end{array}$ & $\begin{array}{l}\text { Tubers/ } \\
\text { corm } \\
\text { shape }\end{array}$ & $\begin{array}{l}\text { Spininess } \\
\text { of roots }\end{array}$ & $\begin{array}{l}\text { Tuber } \\
\text { skin } \\
\text { colour }\end{array}$ & $\begin{array}{l}\text { Flesh } \\
\text { colour }\end{array}$ & $\begin{array}{l}\text { Leaf } \\
\text { length } \\
\text { (cm) }\end{array}$ & $\begin{array}{l}\text { Leaf } \\
\text { width } \\
(\mathrm{cm})\end{array}$ & $\begin{array}{c}\text { No. of } \\
\text { tubers/ } \\
\text { plant }\end{array}$ & $\begin{array}{c}\begin{array}{c}\text { Length } \\
\text { of main } \\
\text { tuber } \\
(\mathrm{cm})\end{array} \\
\end{array}$ & $\begin{array}{c}\text { Width } \\
\text { of main } \\
\text { tuber } \\
(\mathrm{cm}) \\
\end{array}$ & $\begin{array}{c}\text { Yield of } \\
\text { undrground } \\
\text { tuber/plant } \\
(\mathrm{kg})\end{array}$ \\
\hline BD-7838 & HOM-1 & C & $\mathrm{T}$ & C & S & G-0 & Y-W & 13.19 & 12.93 & 2 & 5.4 & 3 & 0.51 \\
\hline BD-7839 & HOM-6 & A & $\mathrm{T}$ & C & M & G-0 & $\mathrm{Y}-\mathrm{W}$ & 20.86 & 14.02 & 6 & 62.5 & 8.3 & 7.60 \\
\hline BD-7842 & HOM-9 & A & $\mathrm{T}$ & C & M & G-0 & $\mathrm{Y}-\mathrm{W}$ & 19.72 & 13.63 & 11 & 37 & 6 & 4.30 \\
\hline BD-7843 & HOM- I I & A & $\mathrm{T}$ & C & $\mathrm{S}$ & G-0 & W & 18.44 & 10.8 & 4 & 43.2 & 6.2 & 1.05 \\
\hline BD-7844 & HOM-13 & A & $\mathrm{T}$ & C & M & G-0 & $\mathrm{P}$ & 15.28 & 9.23 & 5 & 44 & 7 & 2.80 \\
\hline BD-7845 & HOM-17 & A & $\mathrm{T}$ & C & S & G-0 & $\mathrm{Y}-\mathrm{W}$ & 15.63 & 11.59 & 3 & 33.6 & 6 & 1.00 \\
\hline BD-7847 & HOM-20 & A & $\mathrm{T}$ & C & S & G-0 & $\mathrm{Y}-\mathrm{W}$ & 17.83 & 11.21 & 4 & 40 & 7.3 & 1.48 \\
\hline BD-7851 & HOM-29 & A & $\mathrm{T}$ & I & M & G-0 & W & 18.13 & 11.4 & 6 & 25.5 & 14 & 5.20 \\
\hline BD-7852 & HOM-32 & A & $\mathrm{T}$ & C & M & G-0 & W & 19.72 & 12.63 & 4 & 38.5 & 11.5 & 1.80 \\
\hline BD-7853 & HOM-34 & A & $\mathrm{T}$ & C & S & G-0 & Y-W & 11.86 & 6.86 & 2 & 21 & 4.5 & 0.68 \\
\hline BD-7854 & HOM-37 & A & $\mathrm{T}$ & F & M & G-0 & $\mathrm{Y}-\mathrm{W}$ & 18.93 & 13.78 & 3 & 23.1 & 9.3 & 1.30 \\
\hline BD-7855 & HOM-38 & A & $\mathrm{T}$ & C & S & G-0 & Y-W & 20.14 & 13.24 & 12 & 28 & 6.7 & 4.45 \\
\hline BD-7856 & HOM-39 & A & $\mathrm{T}$ & C & D & G-0 & Y-W & 19.06 & 12.86 & 6 & 34.6 & 5.5 & 1.95 \\
\hline BD-7857 & HOM-43 & A & $\mathrm{T}$ & $\mathrm{C}$ & M & G-0 & Y-W & 18.27 & 12.81 & 1 & 25.5 & 7.4 & 0.85 \\
\hline
\end{tabular}


Table 7. Cont'd.

\begin{tabular}{|c|c|c|c|c|c|c|c|c|c|c|c|c|c|}
\hline BD-7858 & HOM-46 & A & $\mathrm{T}$ & $\mathrm{C}$ & $S$ & G-0 & Y-W & 19.4 & 12.87 & 8 & 38 & 8.5 & 4.23 \\
\hline BD-7859 & HOM-47 & A & $\mathrm{T}$ & $\mathrm{C}$ & $\mathrm{D}$ & $\mathrm{R}$ & $\mathrm{W}$ & 13.83 & 10.33 & 2 & 18 & 9.5 & 0.95 \\
\hline BD-7860 & HOM-48 & A & $\mathrm{T}$ & $\mathrm{C}$ & $\mathrm{S}$ & G-0 & $\mathrm{W}$ & 15.06 & 9.07 & 1 & 19.5 & 4.6 & 0.70 \\
\hline BD-7862 & HOM-51 & A & $\mathrm{T}$ & $\mathrm{C}$ & $\mathrm{S}$ & G-0 & Y-W & 17.81 & 12.13 & 11 & 37.5 & 10.3 & 8.40 \\
\hline BD-7863 & HOM-52 & A & $\mathrm{T}$ & $\mathrm{C}$ & $\mathrm{D}$ & $\mathrm{R}$ & Y-W & 14.36 & 9.19 & 6 & 31.6 & 5.8 & 1.75 \\
\hline BD-7864 & HOM-53 & A & $\mathrm{T}$ & $\mathrm{C}$ & $\mathrm{D}$ & $\mathrm{R}$ & $\mathrm{W}$ & 18.22 & 12.4 & 4 & 40 & 7.5 & 1.24 \\
\hline BD-7866 & HOM-57 & A & $\mathrm{T}$ & $\mathrm{C}$ & M & G-0 & Y-W & 22.21 & 15.07 & 18 & 49.9 & 15.5 & 16.45 \\
\hline BD-7867 & HOM-58 & A & $\mathrm{T}$ & $\mathrm{C}$ & $\mathrm{D}$ & G-0 & Y-W & 21.43 & 13.99 & 20 & 41.8 & 9.2 & 16.10 \\
\hline BD-7868 & HOM-59 & A & $\mathrm{T}$ & $\mathrm{C}$ & $\mathrm{D}$ & $\mathrm{R}$ & Y-W & 21.21 & 14.03 & 2 & 35.5 & 7.2 & 0.90 \\
\hline BD-7869 & HOM-60 & A & $\mathrm{T}$ & $\mathrm{C}$ & $\mathrm{S}$ & G-0 & $\mathrm{W}$ & 19.42 & 12.47 & 16 & 24.3 & 5.5 & 2.50 \\
\hline BD-7870 & HOM-61 & A & $\mathrm{T}$ & $\mathrm{C}$ & M & G-0 & Y-W & 19.86 & 12.62 & 12 & 25.5 & 8.5 & 2.90 \\
\hline BD-7874 & HOM-65 & A & $\mathrm{T}$ & $\mathrm{C}$ & M & G-0 & Y-W & 22.23 & 12.93 & 20 & 37.8 & 11 & 14.45 \\
\hline BD-7875 & HOM-66 & A & $\mathrm{T}$ & $\mathrm{C}$ & M & G-0 & $\mathrm{W}$ & 22.14 & 15 & 11 & 45.6 & 13.7 & 14.2 \\
\hline BD-7876 & HOM-67 & A & $\mathrm{T}$ & $\mathrm{C}$ & $\mathrm{D}$ & $\mathrm{R}$ & $\mathrm{P}$ & 18.36 & 13.29 & 2 & 45 & 7.7 & 1.25 \\
\hline BD-7877 & HOM-68 & A & $\mathrm{T}$ & $\mathrm{C}$ & M & G-0 & Y-W & 19.15 & 12.46 & 23 & 26.5 & 8.5 & 5.80 \\
\hline BD-7878 & HOM-69 & A & $\mathrm{T}$ & C & M & G-0 & $\mathrm{W}$ & 19.08 & 12.88 & 26 & 43.2 & 8.2 & 5.60 \\
\hline BD-7879 & HOM-70 & A & $\mathrm{T}$ & C & M & G-0 & Y-W & 23.14 & 14.52 & 30 & 35.4 & 7.5 & 10.60 \\
\hline BD-7880 & HOM-71 & A & $\mathrm{T}$ & $\mathrm{C}$ & S & G-0 & $\mathrm{W}$ & 20.51 & 13.38 & 3 & 15.8 & 7.2 & 0.81 \\
\hline BD-7881 & HOM-72 & A & $\mathrm{T}$ & $0-0$ & M & G-0 & $\mathrm{W}$ & 15.32 & 7.44 & 5 & 37.5 & 10.6 & 3.20 \\
\hline BD-7882 & HOM-73 & A & $\mathrm{T}$ & $\mathrm{F}$ & M & G-0 & $\mathrm{W}$ & 17.66 & 11.7 & 6 & 13.8 & 8.3 & 1.80 \\
\hline
\end{tabular}


Table 7. Cont'd.

\begin{tabular}{|c|c|c|c|c|c|c|c|c|c|c|c|c|c|}
\hline BD-7883 & HOM-74 & A & $\mathrm{T}$ & $\mathrm{C}$ & M & G-0 & Y-W & 18.07 & 10.59 & 11 & 41.7 & 9.4 & 12.55 \\
\hline BD-7884 & HOM-75 & A & $\mathrm{T}$ & $\mathrm{F}$ & M & G-0 & W & 17.47 & 11.24 & 1 & 20.5 & 7.8 & 1.00 \\
\hline BD-7885 & MR-1 & A & $\mathrm{T}$ & $\mathrm{F}$ & S & G-0 & W & 17.22 & 11.46 & 8 & 34.4 & 7.2 & 1.70 \\
\hline BD-7886 & MR-2 & A & $\mathrm{T}$ & $\mathrm{C}$ & S & B & W & 18.56 & 12 & 2 & 16 & 2.5 & 0.52 \\
\hline BD-7887 & MR-3 & A & $\mathrm{T}$ & $0-0$ & S & G-0 & W & 18.16 & 11.71 & 7 & 32.5 & 10.2 & 3.20 \\
\hline BD-7888 & MR-4 & A & $\mathrm{T}$ & $\mathrm{C}$ & M & G-0 & Y-W & 19.3 & 11.74 & 12 & 44.8 & 8.3 & 11.35 \\
\hline BD-7889 & MR-5 & A & $\mathrm{T}$ & $\mathrm{C}$ & S & G-0 & W & 22.39 & 14.7 & 15 & 66.4 & 10.3 & 14.15 \\
\hline BD-7891 & MR-155 & A & $\mathrm{T}$ & $\mathrm{F}$ & M & G-0 & W & 17.14 & 12.11 & 6 & 15.3 & 9.3 & 1.90 \\
\hline BD-7892 & MR-164 & A & $\mathrm{T}$ & $\mathrm{C}$ & $\mathrm{D}$ & G-0 & W & 19.3 & 12.2 & 5 & 16.1 & 8.3 & 1.10 \\
\hline BD-7893 & MRA-4 & A & $\mathrm{T}$ & $\mathrm{C}$ & S & $\mathrm{R}$ & $\mathrm{P}$ & 20.55 & 10.4 & 26 & 16.2 & 7.8 & 4.00 \\
\hline BD-7894 & MRA-20 & A & $\mathrm{T}$ & $\mathrm{F}$ & $\mathrm{s}$ & $\mathrm{R}$ & $\mathrm{P}$ & 19.93 & 8.84 & 14 & 24.5 & 7.5 & 8.40 \\
\hline BD-7895 & MRA-27 & A & $\mathrm{T}$ & $\mathrm{C}$ & M & G-0 & Y-W & 19.96 & 13.3 & 12 & 54.3 & 10.6 & 12.55 \\
\hline
\end{tabular}

Descriptor's classification Table 6. 3. Stem shape at base -4 ridge- $4 \mathrm{R}$ and 7 ridge-7R; 4 . Stem colour- As per Royal Horticultural Society (RHS) Color Chart-Green group 125-143 ABCD and Purplish-Green-PG; 5. Wing colour- Purple group-75-79 ABCD and Green with purple edge-GP; 6. Petiole colour- All green with purple base-GP, All green with purple at both end-GPB and Green-G; 7. Position of leaf- Alternate-A, Opposite-O and Alternate at base and opposite above-AO; 8. Petiole wing colour- Green-G, Green with purple edges-GP and Purple-P; 9. Leaf shape- Cordate-C and Sagittate Long-SL; 10. Distance between lobes- No measurable distance-N and Intermediate distance-1; 11. Aerial tuber- Absent-A and Present-P; 12. Aerial tuber shape- Round-R, Oval-O, Irregular-1, Elongate-E and Flattened-F, 13. Aerial tuber skin colour- Greyed-brown group- 199 ABCD and Brown group- 200 ABCD, 14. Aerial tuber surface texture- Wrinkled-W and Rough-R; 15. Presence of bumps- Absence-A and Present-P.

Descriptor's classifications Table 7. 16. Twining direction- Clockwise (climbing to the left)-C and Anticlockwise (climbing to the right)-A, 17. Type of underground tuber -Tuber-T and Corm-C, 18. Tuber/corm shape- Oval-Oblong-0- 0, Cylindrical-C, Flattened-F and Irregular-I, 19. Spininess of roots- Sparse-S, Moderate-M and Dense-D, 20. Tuber skin colour- Grayed-orange-G-0, Red-R and Black-B, 21. Flesh colour- White-W, YellowishWhite-Y-W, Purple-P 
the 59 germplasm accessions under D. alata exhibited anticlock twining directions (climbing to the right), green types of leaf, leaf margin, and leaf vein, simple leaf type, acute leaf apex shape and presence of wing all over the stem (Table Ic). High phenotypic diversity was observed in petiole colour and aerial tuber. Moderate phenotypic diversity was found in colour of stem and stem wing, distance between leaf lobes and aerial tuber shape. In third experiment (2004-06), the germplasm accessions exhibited elongate to irregular aerial tuber shape with grayed-orange to grayed-brown tuber skin colour and thin to thick skin thickness. The germplasm accessions also showed yellow, yellow-orange to grayed-orange flesh colour, smooth to rough surface texture and absent to present of bumps on aerial tubers. High phenotypic diversity was exhibited in all the characters of aerial tuber except surface texture. (Fig.2 and Table 2,). In second experiment (2002-04), corm to tuber types of underground stem along with oval-oblong, cylindrical, flattened, and irregular shape of tuber were observed among the germplasm accessions. The germplasm accessions exhibited grayed-orange, red to black tuber skin colour with purple, yellow-white to white tuber flesh colour. Low, medium to dense spine on tuber were found. High phenotypic diversity was obtained from spininess on tuber and tuber flesh colour (Fig. 3 and Table 3). From third experiment (2004-06), rhizome, corm to tuber types of under ground stem were exhibited and oval-oblong, cylindrical, flattened, and irregular shape from under ground tuber were observed among the germplasm accessions. Grayed-orange to red tuber skin colour with white, yellowish-white, yellow, yellow-orange, red to purple and low to dense spine on tuber were found among the yam germplasm accessions. In stiff clay soil, pressed and deformed tubers are formed with poor storage quality. Probably for this reason, the same germplasm accessions exhibited different shapes and sizes in different years at PGRC. All the characters exhibited highly significant variations. High phenotypic diversity was found in tuber shape and tuber flesh colour (Table 4). Leaf length, leaf width, and number of tuber per plant ranged from 11.86 to $23.14 \mathrm{~cm}, 6.86$ to $15.79 \mathrm{~cm}$, and 1 to 30 tubers, respectively. The length of main tuber was 5.4 to $66.4 \mathrm{~cm}$ and width was 2.5 to $15.5 \mathrm{~cm}$. The germplasm accessions exhibited 0.51 to $16.45 \mathrm{~kg}$ underground tuber per plant. The maximum CV was found in yield of underground tuber $(98.74 \%)$ and number of tubers per plant $(82.81 \%)$ and minimum in leaf length (12.55\%, Table 5). Qualitative and quantitative performances of individual accession are shown in Table 6 and 7. For maintenance purpose at PGRC, the under ground tubers are harvested and planted at an interval of 2 to3 years (Fig. 4). Performance on the basis of different shapes, sizes and flesh colour of underground tubers, 13 germplasm accessions viz. BD-8892, BD-8895, BD-8899, BD-8917, BD-8918, BD-8919, BD-8926, BD-8927, BD-8935, BD-8940, BD-8941, BD-8946 and BD-8947 were identified as better than the remaining germplasm accessions. One set of these 
germplasm accessions were distributed to Tuber Crop Research Centre of BARI for varietal improvement programme.

The main and conventional method of propagation of all yam species is vegetative multiplication by tubers or their segments, and rhizome cuttings. The tubers and rhizomes survive in the soil until the next rainy season. Tubers can be stored for several months at normal room temperature. The yam germplasm accessions have been maintaining in the field gene bank of PGRC since 1995.

\section{Conclusion}

Fifty nine accessions under Dioscorea alata genotype and one accession under $D$. bulbifera genotype are identified on the basis of stem twining direction, presence of winged, ridges or spines on stem, leaf shape, aerial tuber shape and size, and other qualitative characters.

\section{References}

Ammirato, P.V. 1984. Yams. In: Handbook of Plant Cell Culture. Vol. 3. Crop Species. P.V. Ammirato, D. A. Evans, W. R. Sharp and Y. Yamada (eds.). Macmillan Publishing Company, New York. pp. 327-354.

Chomchalov, N. 1985. Field genebank in South-East Asia. IBPGR Newsletter (IBPGR/ASEAN). 10: 3.

Cowan, J.M. 1928. The Flora of Chakaria Sundarban. Rec. Bot. Surv. India. 11 (2): 197-226.

Heinig, R.L. 1925. List of Plants of Chittagong Collectorate and Hill Tracts. Darjeeling. pp. 71-78.

Hooker, J.D. 1892. Flora of British India. 4: 288-297.

IPGRI, 1997. Descriptors for Yam (Dioscorea spp.). International Plant Genetic Resources Institute (IPGRI), Rome Italy.

Islam M. H., M. O. Islam and M. M. Hoque. 1995. A Report on the Ethnobotanical Survey to Document Local Knowledge on the Uses and Conservation of Taros and Yam. Plant Breeding Division, BARI, Joydebpur, Gazipur, Bangladesh. p. 29.

Islam, M.O., M. M. Haque, M. A Malek, T. Islam and M. Islam. 1997. Plant Genetic Resources Collection, Documentation, Data Management and Information Dissemination: Present Status and Future Strategies. In: Proceedings of a National Workshop on Plant Genetic Resources, G. M. Hossain, R. K. Arora and P.N. Mathur (eds.), 26-29 August, BARC, Dhaka. pp. 256-259.

Jamago, J.M.2000. Morpho-agronomic and molecular diversity of the Philippine mungbean (Vigna radiata L.) germplasm. Master of Science Thesis, University of the Philippines Los Banos, The Philippines.p.174.

Mabberley, D.J. 1997. The Plant Book. Cambridge University Press, The Edinburgh Building, Cambridge C132 2RU, UK. pp.231-232.

Onwueme I. C.1978. The Tropical Tuber Crops Yams, Cassava, Sweet potato and Cocoyams, John Willey and Sons Ltd., Chichister, New York, p. 234. 
Prain, D. 1903. Bengal Plants. Vol. 2. Calcutta, pp. 800-803.

Prain, D. 1903a. Flora of Sundarbans. Rec. Bot. Surv. India. 114: 345.

Rahman, M.A., S. C. Das, M. Yusuf and C.C. Wilcock. 1998. Three new records in the Dioscoreaceae from Bangladesh. Bangladesh J. Plant Taxon. 5 (1): 77-83.

Rahman, M.A., S.C. Das and C.C. Wilcock. 2001. Dioscoreaceae of Bangladesh: Diversity, Distribution and Ethnobotany. Bangladesh J. Plant Taxon. 8 (1): 19-26.

Raizada, M.B. 1941. On the flora of Chittagong. Ind. For. 67: 245-254.

Roxburgh, W. 1814. Hortus Bengalensis. Calcutta, pp. 105.

Roxburgh, W. 1832. Flora Indira. pp. 727-730.

Sinclair, J. 1956. The Flora of Cox's Bazar, East Pakistan, Bull. Bot. Soc. Beng. 9(2): 109.

Thomas, T. A and P. N. Mathur. 1991. Germplasm Evaluation and Utilization. In: Plant Genetic Resources Conservation and Management. R. S. Paroda and R. K. Arora (eds.).Published by the International Board for Plant Genetic Resources, Regional Office for South and Southeast Asia, NBPGR, Pusa Campus, New Delhi 110012, India. pp. 149-18 1.

Yu Li W., K. Shuzhi, C.A.O. Youngsheng and X. Zhang. 1996. A phenotypic diversity analysis of foxtail millet (Setaria italica L.P. Beauv) landrace of Chinese origin. Genetic Resources and Crop Evol. 43: 377-384.

Zaman, S. M. H. 1989. Non-conventional food crops of Bangladesh. In: Plant Breeding in Bangladesh, L. Rahman and A.Q. Shaikh (eds.), Proceedings of the First National Symposium, June 5-7, 1989, BJRI, Dhaka, Bangladesh. pp. 174-187. 